\title{
Intrahepatic cholangiocarcinoma with predominant ductal plate malformation pattern
}

\author{
Ji-Young Choe and Haeryoung Kim \\ Department of Pathology, Seoul National University Bundang Hospital, Seoul National University College of Medicine, Seongnam, \\ Korea
}

Keywords: Cholangiocarcinoma; Ductal plate malformation; Pathology; Liver

\section{INTRODUCTION}

Cholangiocarcinoma arising in the liver can be classified into intrahepatic (or peripheral) cholangiocarcinoma (ICC) or perihilar cholangiocarcinoma, depending on the topographic location of the tumor along the intrahepatic biliary tree.' Grossly, peripheral ICC usually presents as a mass-forming type, while perihilar ICC may present as mass-forming, periductal infiltrating, or intraductal-growth types. Histologically, ICC is heterogeneous: while the majority of ICC present predominantly as a mucin-producing adenocarcinoma, some tumors take the form of a cholangiolocellular carcinoma (CCC), and others demonstrate mixtures of these components within the tumor.

Recently, Nakanuma et al proposed a new subtype of ICCs resembling the histologic features of ductal plate malformation (DPM). ${ }^{2}$ DPM is a developmental anomaly in which the fetal ductal plate persists after birth, and is found mainly in congenital or various acquired hepatobiliary diseases. ${ }^{3}$ ICC with DPM pattern is histologically characterized by irregular and tortuous glandular structures with bridge formation, biliary cell projections into the lumens, and intraluminal tumor cells. ${ }^{2}$ The histopathologic findings of ICC with predominant DPM pattern are discussed, based on the following case which we recently encountered.

\section{CASE}

A 67-year-old male patient was referred to our hospital for further evaluation of a hepatic mass. He complained of general weakness and weight loss during the past 10 months. He was taking medications for diabetes mellitus, hypertension, prostatitis, and atrial fibrillation, and denied any previous intake of alcohol or smoking.

The initial laboratory findings demonstrated elevated serum alkaline phosphatase level (201 IU/L), while all other liver function tests were within normal limits, including aspartate aminotransferase $17 \mathrm{IU} / \mathrm{L}$, alanine aminotransferase $15 \mathrm{IU} / \mathrm{L}$, and total bilirubin $0.8 \mathrm{mg} / \mathrm{dL}$. Serum alpha-fetoprotein and protein induced by vitamin $\mathrm{K}$ absence/antagonist-II levels were $16 \mathrm{ng} / \mathrm{mL}$ and $27 \mathrm{mAU} /$ $\mathrm{mL}$, respectively. Serologic tests for hepatitis B and hepatitis C virus were negative. Contrast-enhanced computed tomography and magnetic resonance images showed a hepatic mass with peripheral enhancement in segment 8 (Fig. 1A, 1B). The center of the tumor remained poorly enhanced. A fluorodeoxyglucose positron emission tomography scan showed isometabolism in the hepatic mass (maximum SUV: 3.0 ) and diffuse hypermetabolism in the multiple bones. The hypermetabolic bone lesions were subsequently confirmed as involvement by multiple myeloma on bone

\section{Abbreviations:}

CCC, cholangiolocellular carcinoma; DPM, ductal plate malformation; ICC, intrahepatic cholangiocarcinoma

\section{Corresponding author : Haeryoung Kim}

Department of Pathology, Seoul National University College of Medicine, Seoul National University Bundang Hospital, 300 Gumi-dong, Bundanggu, Seongnam 463-707, Korea

Tel. +82-31-787-7715, Fax. +82-31-787-4012

E-mail; medannabel@gmail.com 
marrow biopsy, urine/serum protein electrophoresis and immunofixation electrophoresis. The hepatic mass was revealed to be an adenocarcinoma on ultrasonography-guided gun biopsy, and a right anterior sectionectomy of the liver was subsequently performed.

\section{PATHOLOGIC FINDINGS}

Macroscopically, an ill-defined lobulated subcapsular mass, measuring $6.8 \mathrm{~cm}$ in greatest dimension was seen in the resected liver (Fig. 1C). The mass was solid and greenish-white with a hemorrhagic-looking cut surface. The background liver was non-cirrhotic and grossly unremarkable. On histopathologic evaluation, the central portion of tumor was hypocellular with abundant hyalinized fibrous stroma, whereas the peripheral portion was hypercellular with compact tumor glands and less stroma (Fig. 2A). The tumor cells were arranged in glandular structures which were generally larger in the central portion and smaller in the peripheral areas of the tumor. The larger glands were architecturally remark-
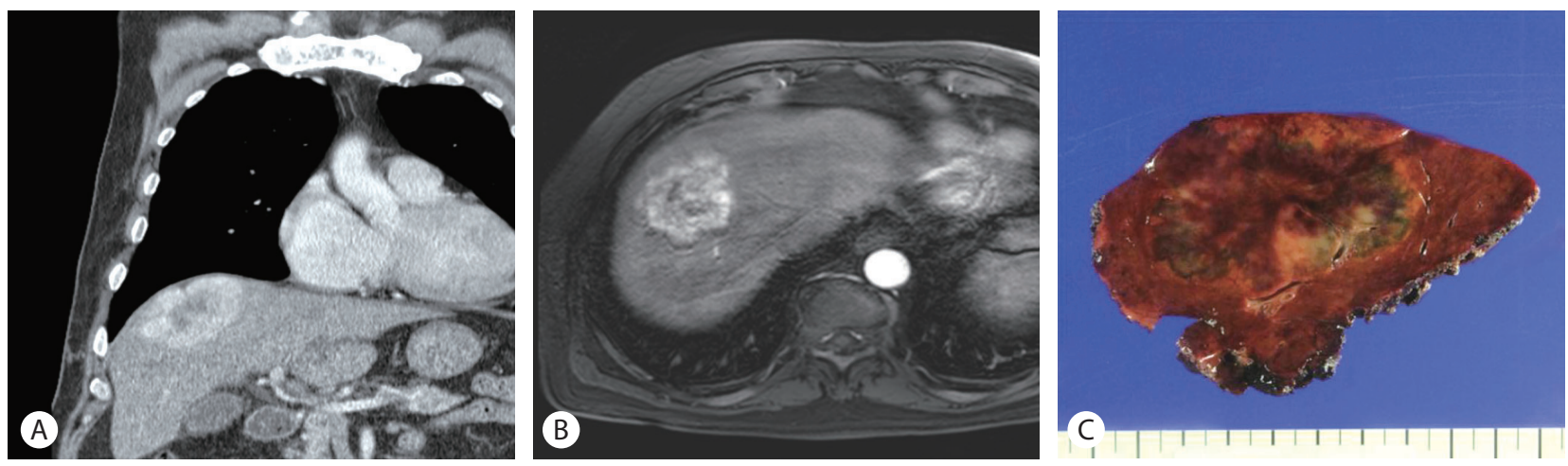

Figure 1. Imaging and gross findings. A coronal image of contrast-enhanced CT (A) and a transverse image of MRI (B) demonstrate a single, lobulated and peripherally enhanced mass in the dome of the right liver. Grossly, the mass is solid and greenish white, with hemorrhagic-looking foci (C).
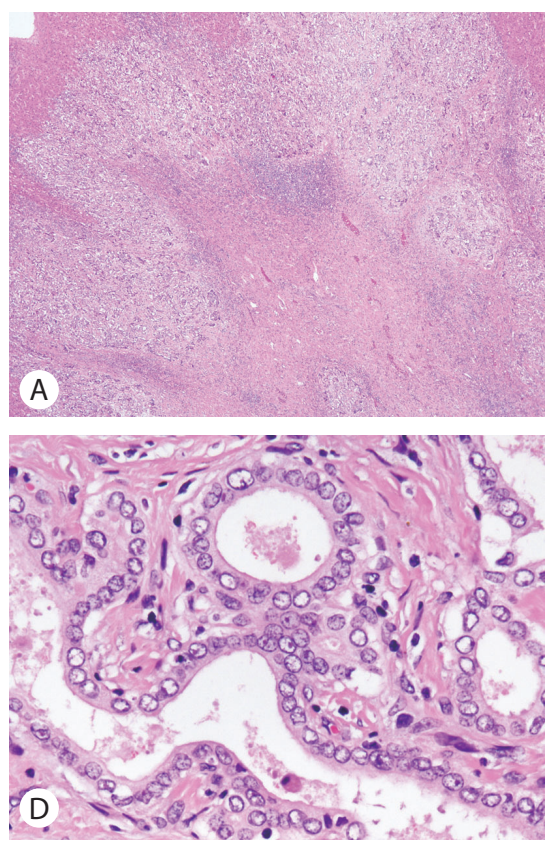
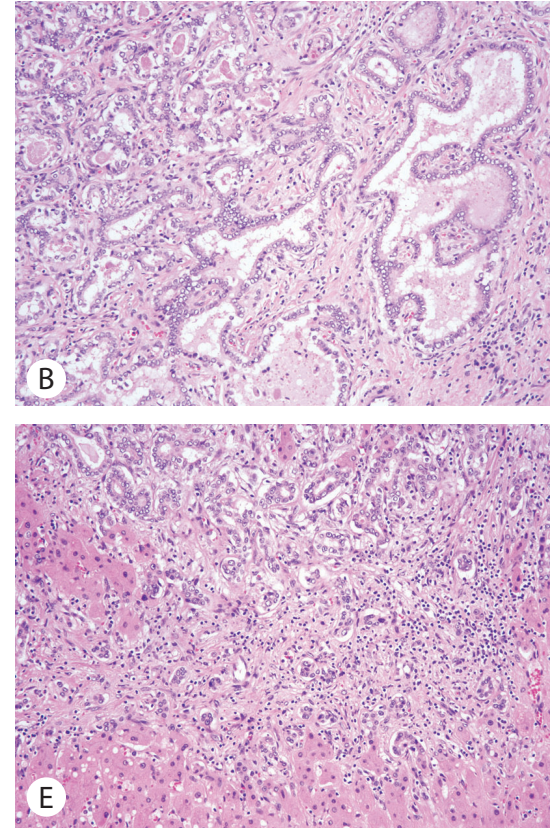
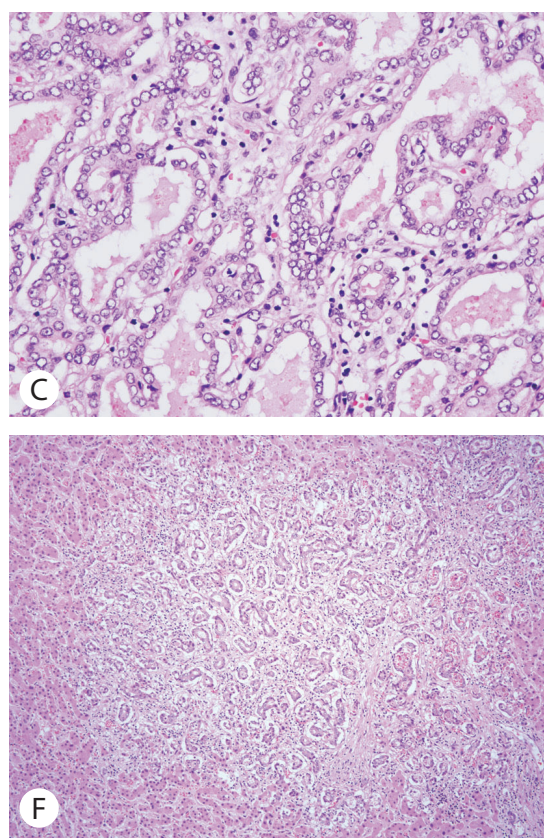

Figure 2. Microscopic findings. (A) The tumor is hypercellular in the peripheral areas, and has a hypocellular central portion with fibrous and hyalinized stroma. $(B, C)$ Variable-sized, irregularly dilated glandular structures are seen, characteristic of a DPM-like pattern. (D) The tumor cells are cuboidal with high nucleocytoplasmic ratios, and round vesicular nuclei. (E, F) The CCC component of the tumor is composed of smaller neoplastic gland-like structures resembling ductular reactions [Hematoxylin-eosin, original magnification $\times 40(A), \times 100(B, E, F), \times 200(C), \times 400(D)$ ]. 

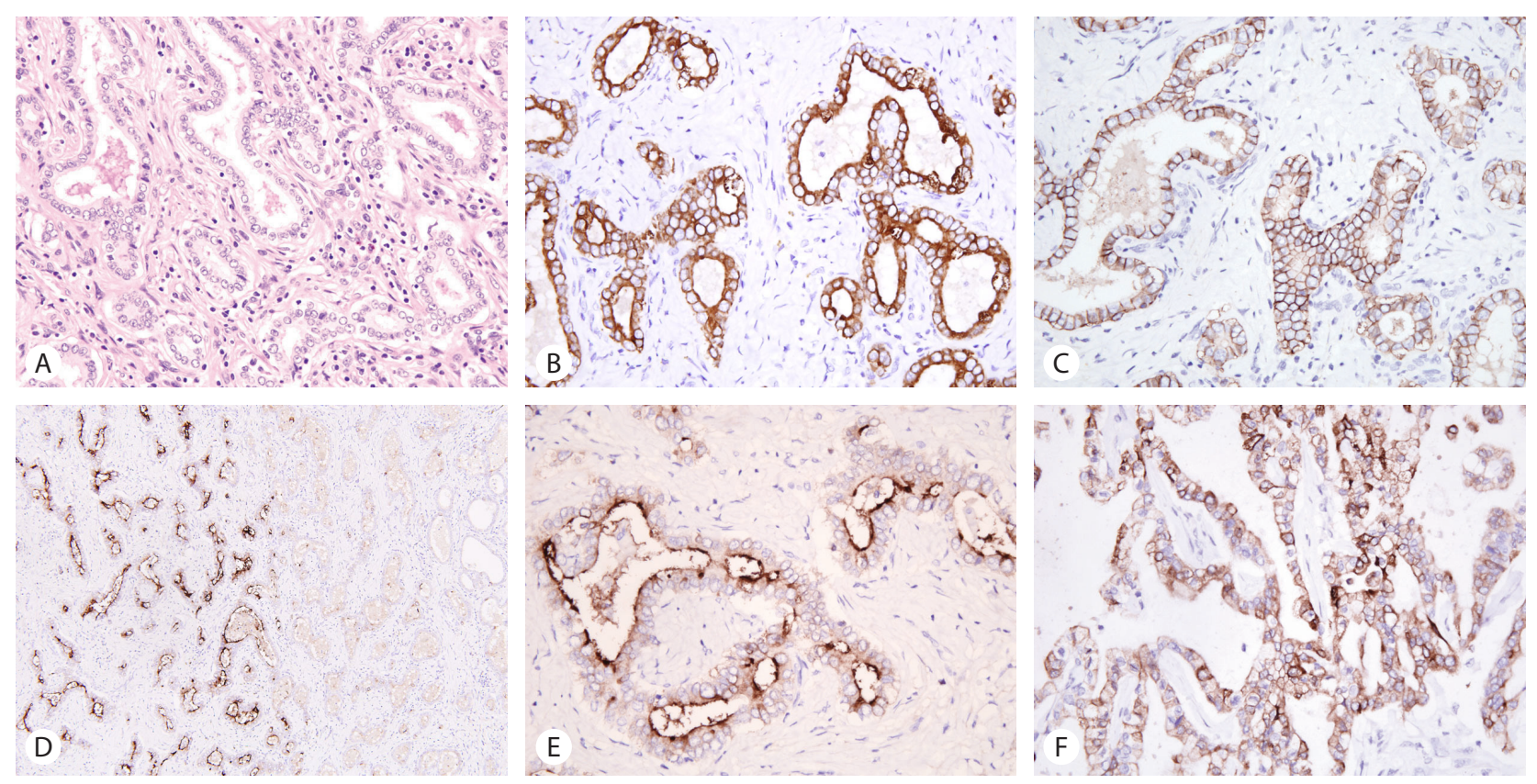

Figure 3. Histochemical and immunohistochemical findings. The tumor is negative for D-PAS (A). Immunohistochemical stains demonstrate cytoplasmic K19 expression (B), membranous EpCAM expression (C), and apical EMA expression (D, E) in the ICC with DPM pattern. An example of membranous EMA expression in a case of mucin-producing cholangiocarcinoma is shown for comparison (F) $[(A, B, C, E, F) \times 200,(D) \times 100]$.

ably similar to DPM: they formed irregular distorted gland-like structures with bridge/island formation and microcystic dilatation (Fig. 2B, 2C). The neoplastic cells were pale eosinophilic, small, and cuboidal or columnar in shape and had high nucleocytoplasmic ratios. The nuclei were small and vesicular, with fine chromatin and small nucleoli, and mitoses were rare (Fig. 2D). Some larger tumor glands were filled with red blood cells, and a few macrophages were seen inside the lumens. The smaller glands in the periphery resembled bile ductules, and cytologic features were similar to those of the DPM-like areas: small cuboidal cells with high nucleocytoplasmic ratio, small vesicular nuclei, fine chromatin and small nucleoli, and resembled bile ductules (Fig. 2E, 2F). This component was histologically compatible with CCC. There was no evidence of mucin production in both the DPM-like and CCC components on diastase-periodic acid Schiff (D-PAS) stain (Fig. 3A). Tumor necrosis was absent. The tumor borders were infiltrative and normal portal tracts were entrapped within the tumor. The non-neoplastic liver showed mild macro- and microvesicular steatosis.

Immunohistochemical stains revealed diffuse cytoplasmic expression of keratin 19 (K19) and basolateral membranous expression of epithelial cell adhesion molecule (EpCAM) in both the DPM-like and CCC components (Fig. 3B, 3C). Epithelial membrane antigen (EMA) was expressed in both components with an apical staining pattern, in contrast to typical mucin-producing cholangiocarcinomas where EMA stain is usually membranous (Fig. 3D-3F). Neural cell adhesion molecule (NCAM, CD56) expression was weak and focal in both components. c-Kit was not expressed in both DPM-like and CCC components, and the tumor was negative for markers of hepatocytic differentiation (HepPar1 and alpha-fetoprotein (AFP)). The Ki-67 labeling index was $<5 \%$.

\section{DISCUSSION}

ICC is a histomorphologically heterogeneous tumor, reflecting the different origins of the tumor cells according to topographic location along the biliary tree. The columnar mucin-producing cholangiocytes - which are the predominant cellular population lining the larger intrahepatic bile ducts - have been suggested to be the origin of mucin-producing cholangiocarcinomas, such as hilar cholangiocarcinomas (Klatskin tumors), perihilar cholangiocarcinomas, and the mucin-producing ICC. At the other end of the biliary tree, the small cuboidal non-mucin-producing cholangiocytes may give rise to CCCs which resemble ductular reactions. ${ }^{3}$ The latter type of cholangiocytes have been postulated to include hepatic progenitor cells, and due to increasing evidence that CCCs may be derived from hepatic progenitor cells, these tumors have 
been re-classified in the latest WHO classification as a subtype of combined hepatocellular-cholangiocarcinomas with stem cell feature. $^{4}$

The recently described ICC with predominant DPM pattern is an interesting variant of ICC that demonstrates large, irregularshaped, tortuous glandular structures resembling fetal ductal plates. ${ }^{2}$ Ductal plates are normally not found in the postnatal liver, and persistence of these structures - referred to as DPM - have been reported in association with congenital hepatic fibrosis, polycystic liver and kidney diseases, congenital biliary atresia, von Meyenburg complex and Caroli's disease. ${ }^{5}$ The mechanisms for the development of this peculiar pattern in ICC is not understood, and further studies would be required to elucidate whether 1) DPM and DPM-like ICC have any common genetic mechanisms, 2) whether some of these tumors may actually arise from a pre-existing DPM, and 3) whether these tumors are a different morphologic manifestation of CCC with extremely dilated glandular structures, pointing to a hepatic progenitor cell origin.

In the recent collective review of 10 cases of ICC with DPM pattern by Nakanuma et al, all 10 cases were associated with areas of CCC, characterized by small anastomosing gland-like structures without distinct lumens and resembling reactive bile ductules. ${ }^{2}$ The immunohistochemical profiles (EpCAM, NCAM, K19 and apical EMA expression) of these tumors and the negativity for mucin in the tumor cells have been reported to be similar for the DPMlike component and the CCC component, suggesting that ICC with DPM pattern may be similar to CCC in that they may retain hepatic progenitor cell features. It is therefore possible that ICC with DPM patterns may also fall under the category of combined hepatocellular and cholangiocarcinoma with stem cell features, along with CCC. This was in fact suggested in another recent case report in which a hepatocellular carcinoma component was found within the tumor, and the tumor cells of the DPM-like component also expressed stem cell markers, such as c-kit and CD34, favoring a hepatic progenitor cell origin for this tumor. ${ }^{6}$

The clinical features of ICC with DPM pattern are still poorly characterized. According to the recent reports, these tumors arise in elderly patients (53-81 years), there is a slight male dominance, and the background liver may be almost normal or show chronic liver disease (viral hepatitis, non-alcoholic steatohepatitis, alcoholic liver disease) - these features are similar to those of ICC. ${ }^{2,6}$ Foci of DPM were seen in the background liver in one recent case re- port, suggesting DPM as a possible precursor lesion for this tumor. ${ }^{6}$ The prognosis of ICC with DPM pattern has not been studied yet. Komuta et al reported that mucin-producing hilar and perihilar cholangiocarcinomas were associated with worse prognosis compared to CCC and mixed ICC (ICCS showing mixed components, including CCC areas and areas of hepatocytic differentiation). ${ }^{3}$ The Ki-67 labeling indices were $<5 \%$ in both DPM-like and CCC components in our case, which is similar to the finding by Nakanuma et al where DPM-like areas showed low Ki-67 labeling indices (mean: $4.5 \%$ ) in contrast to ordinary ICC components (>25\%). It is possible that the presence and the extent of a mucinproducing cholangiocarcinoma component may be the determinant of prognosis for these tumors, although this remains to be characterized in further studies.

\section{Conflicts of Interest}

The authors have no conflicts to disclose.

\section{REFERENCES}

1. Nakanuma Y, Curado MP, Franceschi S, Gores G, Paradis V, Sripa B. Intrahepatic cholangiocarcinoma. In: Bosman FT, Carneiro $F$, Hruban RH, Theise ND, eds. WHO classification of tumours of the digestive system. 4th ed. Lyon: IARC, 2010:217-224.

2. Nakanuma Y, Sato Y, Ikeda H, Harada K, Kobayashi M, Sano K, et al. Intrahepatic cholangiocarcinoma with predominant "ductal plate malformation" pattern: a new subtype. Am J Surg Pathol 2012;36:1629-1635.

3. Komuta M, Govaere O, Vandecaveye V, Akiba J, Van Steenbergen W, Verslype C, et al. Histological diversity in cholangiocellular carcinoma reflects the different cholangiocyte phenotypes. Hepatology 2012;55:1876-1888.

4. Theise ND, Nakashima O, Park YN, Nakanuma Y. Combined hepatocellular-cholangiocarcinoma. In: Bosman FT, Carneiro F, Hruban $\mathrm{RH}$, Theise ND, eds. WHO classification of tumours of the digestive system. 4th ed. Lyon: IARC, 2010:225-227.

5. Awasthi A, Das A, Srinivasan R, Joshi K. Morphological and immunohistochemical analysis of ductal plate malformation: correlation with fetal liver. Histopathology 2004;45:260-267.

6. Terada T. Combined hepatocellular-cholangiocarcinoma with stem cell features, ductal plate malformation subtype: a case report and proposal of a new subtype. Int J Clin Exp Pathol 2013;6:737-748. 